\title{
Decision Trees Work Better Than Feed-Forward Back-Prop Neural Nets for A Specific Class of Problems*
}

\author{
Xiaomei Liu, Kevin W. Bowyer \\ Dept. of Computer Science \& Engineering \\ University of Notre Dame \\ Notre Dame, IN 46556 U.S.A. \\ \{xliu5, kwb\}@cse.nd.edu
}

\author{
Lawrence O. Hall \\ Dept. of Computer Science \& Engineering \\ University of South Florida \\ Tampa, FL 33620, U.S.A. \\ hall@csee.usf.edu
}

\begin{abstract}
Feed forward, back propagation neural networks are known to be universal approximators in a certain theoretical sense. They can be time-consuming to train and require significant parameter tuning. Decision trees are generally faster and simpler to train, but are widely assumed to not offer predictive accuracy as good as feed forward, back propagation neural networks. We have noticed in previous work that decision trees tended to outperform feed forward, back propagation neural networks on a certain dataset. We provide a description of a class of problems that are extremely difficult for feed forward, back propagation neural networks but relatively simple for Decision trees. Experiments with synthetic datasets illustrate the class of problems. The importance of this result lies in making decisions about when to employ what type of classifier in practice.
\end{abstract}

Keywords: Neural Networks, Decision Trees, Performance Evaluation, Feature Space.

\section{Introduction}

Feed forward back propagation (FFBP) neural networks $(\mathrm{NN})$ are a very popular tool for pattern classification. They are known to be fully general in the sense being universal approximators [4], [8]. Put simply, there are theoretical results that say that any specified decision boundary in feature space can be approximated arbitrarily closely by some FFBP NN. However, FFBP NNs are generally time-consuming to train and various parameters of the training process will need to be set appropriately in order to obtain the best performance. Again, put simply, the theory says that the "right" NN exists, but there is no direct guidance on how to find it.

Decision trees (DTs) are generally fast and simple to train. The one significant parameter to adjust in training a DT is the degree of pruning [11]. DTs are also widely assumed not to offer accuracy as good as what may be obtained with NNs. However, we have noticed in some previous work that DTs tended to provide better predictive accuracy than FFBP NNs on a certain dataset - the "Forest Cover Type" dataset [3].

\footnotetext{
${ }^{*}$ 0-7803-8566-7/04/\$20.00 C) 2004 IEEE.
}

Based on some experiments with this dataset, we conjecture a class of problems for which DTs generally will work much better than FFBP NNs. We also generate some synthetic datasets to illustrate this class of problems. The general characteristics of this class of problems is that some minority classes are distributed in small regions that are surrounded and overlapped with majority classes. The results presented here are important because they show that there are classes of problems for which a standard DT can be generally expected to achieve better performance than a standard FFBP NN. This suggests that there may be ways of examining the structure of a problem to determine if it is well-suited to solution by NNs.

The remaining parts of this paper are organized as following. In section 2, we will introduce the experiments on the forest covertype data set. In section 3, we will describe how we generated the synthetic data sets and the experiments on the synthetic data sets. Section 4 is the discussion and conclusion.

\section{Experiments on Forest Cover}

The forest covertype data set [3] was obtained from the UCI collection [1]. There are 581,012 instances in the data set. Each instance is described by 54 features: 7 continuous features, 3 discrete features ( 0 to 255), and 44 binary features. The distribution of the seven classes in the data set is shown in Table 1. In our experiments, we followed the train/test split used in [10]. We randomly chose 149,982 records for the training set. The remaining 431,030 records were used for the testing set. The class distribution in the training set and testing set were similar.

\subsection{Basic Experiments}

We trained a FFBP NN on 149,982 training examples. There were 54 input units, 7 output units, and one hidden layer of 30 hidden units. As with the default setting of the software usfquickprop [5], the number of hidden units used in the FFBP NN was the average of the number of the input nodes and the number of the output nodes, which is also the 
Table 1: Class distribution of forest covertype (from [3]).

\begin{tabular}{|l|c|c|}
\hline $\begin{array}{l}\text { CLASS } \\
\text { LABEL }\end{array}$ & $\begin{array}{c}\text { NUMBER OF } \\
\text { INSTANCES }\end{array}$ & PERCENT \\
\hline 1 & 211,840 & $36.5 \%$ \\
\hline 2 & 283,301 & $48.8 \%$ \\
\hline 3 & 35,754 & $6.2 \%$ \\
\hline 4 & 2,747 & $0.5 \%$ \\
\hline 5 & 9,493 & $1.6 \%$ \\
\hline 6 & 17,367 & $3.0 \%$ \\
\hline 7 & 20,510 & $3.5 \%$ \\
\hline
\end{tabular}

default setting used in weka [2]. The input features were normalized linearly into the interval $[0,1]$. The initial weights were randomly generated in the interval $[-0.7,+0.7]$. We used a learning rate of 0.55 , a momentum of 0.9 , and batch training. We trained the FFBP NN for 5,000 epochs, and tested it after each epoch. The best FFBP NN accuracy that we obtained on the testing set was $75.19 \%$, which was obtained at epoch 1,034, while the accuracy of one decision tree (either unpruned or default pruned) was $90.0 \%$. The decision tree greatly outperformed the FFBP NN.

\subsection{Decreasing the Number of Features}

We first suspected that the FFBP NN's lower accuracy was because there were too many binary features. So we converted the problem into a 10 -feature problem by simply dropping the 44 binary features.

We did the experiments using a plain FFBP NN with 10 input units, 7 output units, and one hidden layer of 8 hidden units. The other parameter settings were the same as in the last subsection. We trained the FFBP NN for 5,000 epochs, and tested it after each epoch. The best accuracy on the testing set was $70.65 \%$, which first occured at epoch 2,919 . The unpruned DT's accuracy was $85.5 \%$, and the default pruned DT's accuracy was $85.6 \%$. Thus we concluded that the presence or absence of binary features is not a significant factor in the relative performance of the DT and NN. The performance of both the $\mathrm{NN}$ and the DT drop by about $4.5 \%$ when the 44 binary features are removed from the data set.

We further simplified this problem by dropping the three discrete features and using only the 7 continuous features. The 7 continuous features are features 1 to 6 , and feature 10. For the FFBP NN, we used 7 input units, 7 output units, and one hidden layer of 7 hidden units. The best accuracy of the FFBP NN within the first 1,000 epochs was 69.84\%, which first occured at epoch 237 . The unpruned DT's accuracy was $85.8 \%$, and the default pruned DT's accuracy was $86.0 \%$. Thus we concluded that the presence or absence of the discrete features is not a significant factor in the relative performance of the DT and NN, or even in the absolute performance of the two classifiers.

As shown in the first three rows in Table 2, the accuracy difference between the default pruned DT and FFBP NN increased when we used a smaller number of features. If this
Table 2: Accuracy of using different numbers of features.

\begin{tabular}{|l|c|c|c|}
\hline $\begin{array}{l}\text { NUMBER OF } \\
\text { FEATURES }\end{array}$ & $\begin{array}{c}\text { DEFAULT } \\
\text { PRUNED DT }\end{array}$ & FFBP NN & DIFF \\
\hline 54 & $90.0 \%$ & $75.1 \%$ & $14.9 \%$ \\
\hline 10 & $85.6 \%$ & $70.65 \%$ & $15.18 \%$ \\
\hline 7 & $86.0 \%$ & $69.84 \%$ & $16.16 \%$ \\
\hline 2 & $53.3 \%$ & $50.19 \%$ & $3.11 \%$ \\
\hline
\end{tabular}

trend continues, when we decrease the number of features to 2 , we could plot the results and possibly visualize the cause of the difference between FFBP NN and DT performance.

We decreased the number of features from 7 to 3 in the following way. We excluded one feature at a time. When trying to decrease the number of features from $n$ to $n-1$, we tried all $n$ groups of (n-1) features. For each (n-1)-feature setting, we built a FFBP NN and DT. We chose the (n-1) features with the highest DT accuracy. The order of the features being excluded was: $3,5,4,2$.

When decreasing the number of features from 3 to 2 , we tried all 3 possible 2 -feature settings, choosing the one with the largest accuracy difference between the default pruned DT and the FFBP NN. The 2 features chosen were feature 6 and feature 10 . When using only these 2 features, the FFBP NN's best accuracy was $50.19 \%$ with training for 1,000 epochs, the unpruned DT's accuracy was $52.3 \%$, and the default pruned DT's accuracy was $53.3 \%$. The performance of both the DT and the NN is greatly reduced when using only two features, and the gap between them is small, but the DT still performs better than the FFBP NN. The prediction results are shown in Figure 1 and Figure 2. The FFBP NN could only predict the three majority classes, while the DT could predict all 7 classes.

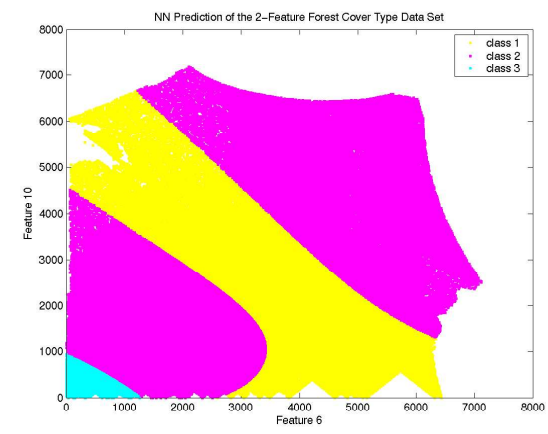

Figure 1: FFBP NN prediction of the 2-dimensional covertype data set (using 4 hidden units).

In order to improve the FFBP NN's accuracy, we increased the number of hidden units from 4 to 80 . After training for 10,000 epochs, the 80-hidden-unit FFBP NN's prediction results are shown in Figure 3. It could still only predict the three majority classes, although it had a smoother and more complex boundary. 


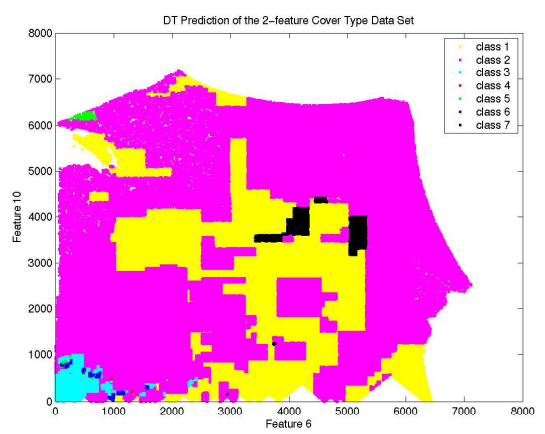

Figure 2: Unpruned DT prediction of the 2-dimensional covertype data set.

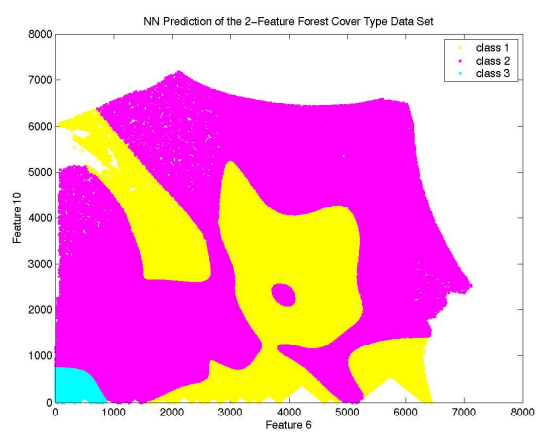

Figure 3: FFBP NN prediction of the 2-dimensional covertype data set (using 80 hidden units).

As listed in Table 1, the three majority classes (class 1, 2, 3) account for $90 \%$ of all the data. The other four minority classes are surrounded and overlapped by the three majority classes. Since we only used two features, the overlapping of different classes is somewhat expected. We could not necessarily conclude that this is the reason for the relatively lower perfermance of the FFBP NN on the original 54 feature data set. However, on the specific two dimensional version of the problem, the DT still worked better than the FFBP NN. This gave us some hints to formulate a class of problems that favor the DT.

\section{Experiments on Synthetic Sets}

From our experiments on the Forest CoverType Data Set, we conjecture a class of problems that are relatively easy for DTs to solve but much more difficult for FFBP NNs to solve. The key feature of this class of problems is that some minority classes are distributed over relatively small regions of feature space and surrounded and overlapped by some majority classes. We generated some synthetic data sets to represent various instances of this situation. We simplified the problem to use just two classes. One minority class is surrounded and overlapped by another majority class.
Table 3: DT results for the basic experiments, and for islands with size $0.02 \times 0.02$ (in parentheses).

\begin{tabular}{|l|cr|}
\hline ACTUAL & \multicolumn{2}{|c|}{ PREDICTED CLASS } \\
CLASS & CLASS 1 & CLASS 2 \\
\hline CLASS 1 & 499,863 & 137 \\
& $(499,374)$ & $(626)$ \\
\hline CLASS 2 & 12 & 288 \\
& $(8)$ & $(1,192)$ \\
\hline
\end{tabular}

\subsection{Basic Experiments}

For each object there are two features $[\mathrm{x}, \mathrm{y}]$. As shown in Fig. 4, there are two classes: class 1 and class 2. Class 1 is the majority class. The feature space is $[0,1] \times[0,1]$. There are 500,000 elements of class 1 randomly distributed over the feature space. There are three small "islands" in which minority class elements are distributed: $[0.24,0.25] \times$ $[0.24,0.25],[0.49,0.50] \times[0.49,0.50]$, and $[0.74,0.75] \times$ $[0.74,0.75]$. Each island is of the size $0.01 \times 0.01$. There are 100 elements of class 2 randomly distributed in each island. The average number of class 1 elements in each island of size $0.01 \times 0.01$ is 50 . So for each island, the expected ratio of class 2 to class 1 is $2: 1$.

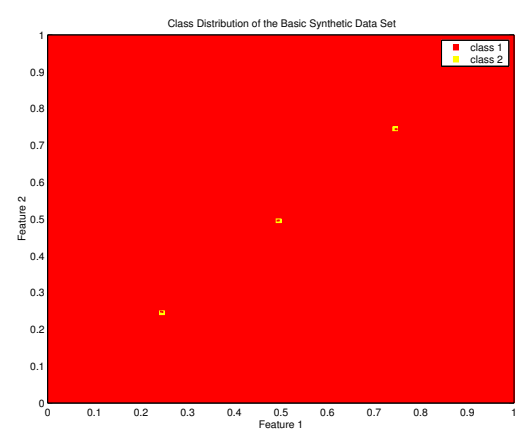

Figure 4: Class Distribution of the basic synthetic data

We generated the training data and the testing data in the same way with different random seeds. Together, there are 500,300 elements in the training data set and in the testing data set.

We used a FFBP NN with 2 input units, 2 output units, and 1 hidden layer of 80 hidden units. We trained the FFBP NN for 10,000 epochs with batch training. The FFBP NN predicted all the elements in the testing set as class 1 . So the accuracy was 500,000/500,300 $=99.94 \%$.

We did experiments on the same data sets with one C4.5 DT. Identical results were obtained whether using a default pruned DT or an unpruned DT. The results are shown in Table 3. The prediction accuracy was $(499,863+288) / 500,300=99.97 \%$. The DT clearly performs better than the FFBP NN by being able to predict better for the minority class. 
Thus the general description of a problem that is challenging to FFBP NNs seems clear. When there is a minority class that is distributed in small isolated regions of feature space, the BP learning mechanism seems to have great trouble "finding" the regions.

\subsection{Different Size of Islands}

In these experiments, we tried to increase the size of each island from $0.01 \times 0.01$ to $0.02 \times 0.02$. There are still 500,000 elements of class 1 randomly distributed in the total area $[0,1] \times[0,1]$. There are 3 islands: $[0.24,0.26] \times[0.24,0.26]$, $[0.49,0.51] \times[0.49,0.51]$, and $[0.74,0.76] \times[0.74,0.76]$. Each island is of the size $0.02 \times 0.02$. There are 400 elements of class 2 randomly distributed in each island. So the expected number ratio of class 2 to class 1 for each island is still 2:1.

We generated the training data set and the testing data set in the same way with different random seed. There are 501,200 elements each in the training data set and in the testing data set.

We used a FFBP NN with 2 input units, 2 output units, and 1 hidden layer of 80 hidden units. We trained the FFBP $\mathrm{NN}$ for 10,000 epochs with batch training. The FFBP NN still predicted all the elements in the testing set as class 1 . So the accuracy was 500,000/501,200 $=99.76 \%$. Compared with the last subsection, the decrease of FFBP NN accuracy was due to the increase of the number of class 2 examples in the testing data set.

We did experiments on the same data sets with one unpruned C4.5 DT. The DT prediction results are shown in Table 3 . The accuracy was $(499,374+1192) / 501,200=99.87 \%$. Compared with the last subsection, the decrease of the accuracy was because more elements of class 1 would fall into the region of class 2 with a larger island. The default pruned DT worked exactly the same as the unpruned C4.5 DT.

\subsection{Different Numbers of Islands}

In these experiments, we tried to increase the number of islands to 9: $[0.10,0.11] \times[0.10,0.11],[0.20,0.21] \times$ $[0.20,0.21], \ldots,[0.90,0.91] \times[0.90,0.91]$. For each island, the ratio of class 2 and class 1 is $2: 1$.

The FFBP NN still predicted all the elements in the testing set as class 1 . So the accuracy was $500,000 / 500,900=$ $99.82 \%$. The unpruned DT's accuracy was $99.92 \%$, and the default pruned DT had the same accuracy as the unpruned one.

\subsection{Different Ratio Between Classes}

In these experiments, we used the original 3 islands, each with size of $0.01 \times 0.01$. But for each island, we tried to increase the element ratio between class 2 and class 1 . We tried the ratios $4: 1$ and $8: 1$. For each case, the FFBP NN predicts all the elements in the testing set as class 1 . The DT tree can still separate the two classes. Table 4 shows the DT and FFBP NN prediction accuracies on the synthetic data sets with different class distribution ratios. By increasing the
Table 4: Accuracies with different class 2 to 1 ratios.

\begin{tabular}{|l|c|c|}
\hline RATIO & DT & FFBP NN \\
\hline $2: 1$ & $99.97 \%$ & $99.94 \%$ \\
\hline $4: 1$ & $99.97 \%$ & $99.88 \%$ \\
\hline $8: 1$ & $99.97 \%$ & $99.76 \%$ \\
\hline
\end{tabular}

Table 5: Accuracies with different size islands.

\begin{tabular}{|l|c|c|c|c|}
\hline SIZE & DT & FFBP & RPROP & $\begin{array}{r}\text { CAS. } \\
\text { CORR. }\end{array}$ \\
\hline $0.01 \times 0.01$ & $99.97 \%$ & $99.94 \%$ & $99.94 \%$ & $99.95 \%$ \\
\hline $0.02 \times 0.02$ & $99.87 \%$ & $99.76 \%$ & $99.76 \%$ & $99.79 \%$ \\
\hline
\end{tabular}

ratio between class 2 and class 1 for each island from 2:1 to $8: 1$, both the unpruned DTs and the default pruned DTs prediction accuracies did not change. Although the FFBP NN prediction accuracies changed, it was only the result of the change in the distribution of the two classes. The FFBP NNs predicted all elements as class 1 .

\subsection{Other FFBP NNs}

In these experiments, we tried to use Rprop [12] and Cascade Correlation NN [7]. The algorithm Rprop was created from the original feed forward and back propagation NN. The main idea of Rprop is that different connections between neurons should have different learning rates, and the learning rates should be updated dynamically. The algorithm we implemented was an updated version [9] of Rprop. We used a Rprop NN with one hidden layer of 80 hidden units. We trained the Rprop NN for 100,000 epochs.

The Cascade Correlation NN is a self configuring feed forward and back propagation neural network. The software we used, usfcascor, which was modified from the $\mathrm{C}$ port of Scott Fahlman's Cascade Correlation Neural Network [6] by the researchers at University of South Florida. We used the following parameters for the cascade correlation NN: the maximum input epoch $=1000$, the maximum output epoch $=$ 1000 , and the maximum of units $=80$.

\subsubsection{SIZE}

Table 5 shows the accuracies of different classifiers with different sizes of islands. There are a total of 3 islands. For each island, the ratio of class 2 and class 1 is $2: 1$.

Compared with plain FFBP NN, Rprop did not show any advantage here. Rprop predicted all elements as class 1, and had the same accuracy as the plain FFBP NN. Cascade Correlation NN had a little better accuracy than the other two NNs in predicting both classes, but the accuracy was still worse than the DT.

\subsubsection{NUMBER}

Table 6 shows the accuracies of different classifiers with different numbers of islands. Each island is of size $0.01 \times$ 0.01 . For each island, the ratio of class 2 and class 1 is $2: 1$. 
Table 6: Accuracies with different numbers of islands.

\begin{tabular}{|l|c|c|c|r|}
\hline $\begin{array}{l}\text { NUMBER } \\
\text { ISLANDS }\end{array}$ & DT & $\begin{array}{c}\text { PLAIN } \\
\text { FFBP }\end{array}$ & RPROP & $\begin{array}{r}\text { CAS. } \\
\text { CORR. }\end{array}$ \\
\hline 3 & $99.97 \%$ & $99.94 \%$ & $99.94 \%$ & $99.95 \%$ \\
\hline 9 & $99.91 \%$ & $99.82 \%$ & $99.82 \%$ & $99.83 \%$ \\
\hline
\end{tabular}

Table 7: Results for 9 islands of size $0.01 \times 0.01$; DT results above, $\mathrm{CC}$ NN results below (in parentheses)

\begin{tabular}{|l|cr|}
\hline ACTUAL & \multicolumn{2}{|c|}{ PREDICTED CLASS } \\
CLASS & CLASS 1 & CLASS 2 \\
\hline CLASS 1 & 499,559 & 441 \\
\hline & $(499,825)$ & $(175)$ \\
\hline CLASS 2 & 33 & 867 \\
\hline & $(709)$ & $(191)$ \\
\hline
\end{tabular}

Compared with plain FFBP NN, Rprop still did not show any advantage here. Rprop predicted all elements as class 1 , and had the same accuracy as the plain FFBP NN. The Cascade Correlation NN had a little better accuracy than the other two NNs, but it was still worse than the DT. For the data sets with 9 islands, the DT and CC NN prediction results are shown in Table 7.

\subsubsection{RATIO}

Table 8 shows the accuracies of different classifiers with different ratios of classes distribution on each of the 3 islands with size $0.01 \times 0.01$.

When the ratios are 2:1 and 4:1, the Rprop predicted all elements as class 1 , and had the same accuracy as the plain FFBP NN. When the ratio is 8:1, Rprop can differentiate the two classes, and thus it had a little better accuracy than the plain FFBP NN. For all the ratios, the cascade correlation NN had a little better accuracy than the other two NNs, but it was still worse than the DT.

\subsection{FURTHER EXPERIMENTS}

We tried further experiments with two classes that have the same number of elements, and there is one island of each in the other's territory. The feature space is $[0,1] \times[0,1]$. There were around 400,000 of class one in the top half of the space and around 400,000 of class two in the bottom half of the space. Then add one island at $[0.24,0.26] \times[0.24,0.26]$. Add another 1,280 class 2 elements at this island. The island is in the top half where class one predominates. In the island, there is a 4-to-1 ratio in favor of class two. And add a similar island in the bottom half where class two predominates. There are two cases.

Case I: The upper space and the lower space are separated by a diagonal line.

In the ideal classification, the two islands will be classified as the dominating class in each island. For the space outside of the islands, the upper space will be predicted as class one, and the lower space will be predicted as class two. Table 9
Table 8: Accuracies with different class 2 to 1 ratios.

\begin{tabular}{|l|c|c|c|c|}
\hline RATIO & DT & FFBP & RPROP & $\begin{array}{r}\text { CAS. } \\
\text { CORR. }\end{array}$ \\
\hline $2: 1$ & $99.97 \%$ & $99.94 \%$ & $99.94 \%$ & $99.95 \%$ \\
\hline $4: 1$ & $99.97 \%$ & $99.88 \%$ & $99.88 \%$ & $99.90 \%$ \\
\hline $8: 1$ & $99.97 \%$ & $99.76 \%$ & $99.79 \%$ & $99.91 \%$ \\
\hline
\end{tabular}

Table 9: Accuracies with diagonal line separating classes, and with horizontal line (in parentheses).

\begin{tabular}{|c|c|c|c|r|}
\hline & IDEAL & DT & CC & FFBP \\
\hline OVERALL & $99.92 \%$ & $99.80 \%$ & $99.85 \%$ & $99.66 \%$ \\
\hline & $(99.92)$ & $(99.87)$ & $(99.80)$ & $(99.68)$ \\
\hline ISLAND & $80.00 \%$ & $79.91 \%$ & $72.03 \%$ & $20.00 \%$ \\
\hline & $(80.15)$ & $(79.18)$ & $(63.53)$ & $(19.85)$ \\
\hline
\end{tabular}

shows the overall accuracy and island accuracy (the accuracy on the elements within the two islands) of the ideal situation, DT, CC (Cascade Correlation NN), and FFBP NN. Table 10 shows the details of the classes distribution and prediction in the case where the classes are separated by a diagonal boundary, and Table 11 shows the details in the case of a horizontal bounday.

Our experimental result shows that the $\mathrm{CC} \mathrm{NN}$ is better able to adapt to the requirements of this problem than the plain FFBP NN. It also shows that DT problems with approximating a non-axis-parallel boundary can obscure the DT's ability to better find the minority class islands. It confirms that the universal approximation about NNs has some value, but it still also points out that the "right" NN architecture can be very hard to find.

\section{Summary and Discussion}

Based on some experiments with the Forest CoverType Data Set, we conjectured a class of problems that are extremely difficult for FFBP NNs but relatively simple for DTs. The features of such problems are that some minority classes are surrounded and overlapped by some majority classes. We generated synthetic data sets with the above description. In our experiments on the synthetic data sets, we varied the size and the number of the islands for the minority class. We also varied the ratio of the minority class and the majority class for each island. We tried a plain feed forward back propagation NN, a Rprop NN, and a cascade correlation NN. With all of these experiments, the DT performed better than each kind of FFBP NN. We further did the experiments of using two classes with the same number of elements, where there is one island of each in the other's territories. For the case of separating the two classes by a diagonal boundary, the DT worked a little worse than the CC NN, but better than the plain FFBP NNs. But the DT worked much faster than the CC NN. For the case of separating the two classes by a horizontal boundary, the DT worked best. The advantages of 
Table 10: The classes distribution and prediction results for case I.

\begin{tabular}{|c|c|c|c|c|c|c|c|}
\hline & \multirow{2}{*}{$\begin{array}{l}\text { \# OF EXAMPLES } \\
\text { IN TESTING SET }\end{array}$} & \multicolumn{2}{|c|}{ DT } & \multicolumn{2}{|c|}{ CC NN } & \multicolumn{2}{|c|}{ FFBP NN } \\
\hline & & CLASS 1 & CLASS 2 & CLASS1 & CLASS 2 & CLASS 1 & CLASS 2 \\
\hline $\begin{array}{l}\text { IN THE UPPER SPACE } \\
\text { OUTSIDE OF ISLANDS }\end{array}$ & 400,418 class 1 & 399,924 & 494 & 400,228 & 190 & 400,329 & 89 \\
\hline IN THE UPPER ISLAND & $\begin{array}{l}326 \text { class } 1 \\
1,280 \text { class } 2\end{array}$ & $\begin{array}{l}0 \\
0\end{array}$ & $\begin{array}{c}326 \\
1,280\end{array}$ & $\begin{array}{c}49 \\
233\end{array}$ & $\begin{array}{c}277 \\
1,047\end{array}$ & $\begin{array}{c}326 \\
1,280\end{array}$ & $\begin{array}{l}0 \\
0\end{array}$ \\
\hline $\begin{array}{l}\text { IN THE LOWER SPACE } \\
\text { OUTSIDE OF ISLANDS }\end{array}$ & 398,942 class 2 & 469 & 398,473 & 142 & 398,800 & 45 & 398,897 \\
\hline IN THE LOWER ISLAND & $\begin{array}{l}1,280 \text { class } 1 \\
314 \text { class } 2\end{array}$ & $\begin{array}{c}1,277 \\
314\end{array}$ & $\begin{array}{l}3 \\
0\end{array}$ & $\begin{array}{c}1,184 \\
289\end{array}$ & $\begin{array}{l}96 \\
25\end{array}$ & $\begin{array}{l}0 \\
0\end{array}$ & $\begin{array}{l}1,280 \\
314\end{array}$ \\
\hline
\end{tabular}

Table 11: The classes distribution and prediction results for case II.

\begin{tabular}{|c|c|cc|cc|cc|}
\hline & \# OF EXAMPLES & \multicolumn{2}{|c|}{ DT } & \multicolumn{2}{c|}{ CC NN } & \multicolumn{2}{c|}{ FFBP NN } \\
& IN THE TESTING SET & CLASS 1 & CLASS 2 & CLASS1 & CLASS 2 & CLASS 1 & CLASS 2 \\
\hline IN THE UPPER SPACE & \multirow{2}{*}{399,369 class 1 } & \multirow{2}{*}{398,957} & 412 & 399,107 & 262 & \multirow{2}{*}{399,352} \\
OUTSIDE OF ISLANDS & 326 class 1 & 0 & 330 & 114 & 216 & 330 \\
\hline IN THE UPPER ISLAND & 1,280 class 2 & 0 & 1,280 & 463 & 827 & 1,280 & 0 \\
\hline IN THE LOWER SPACE & 399,997 class 2 & 1 & 399,996 & 197 & 399,800 & 0 & 399,997 \\
OUTSIDE OF ISLANDS & 1,280 class 1 & 1,280 & 0 & 1,029 & 251 & 0 & 1,280 \\
\hline IN THE LOWER ISLAND & 304 class 2 & 303 & 1 & 245 & 59 & 0 & 304 \\
\hline
\end{tabular}

DT for this class of problem lies in both higher accuracy and more efficiency. It shows that we successfully formulated a kind of problem for which DTs are more useful than FFBP NNs. The importance of this result lies in making decisions on when to employ what type of classifier in practice. All our experiments on the NNs focused on FFBP NNs. Rprop is an improved version of the plain FFBP, and Correlation Cascade is a kind of self configuration FFBP NNs. We are going to do experiments on some other kinds of NNs, such as Radial Basis Functions, in the future.

\section{Acknowledgments}

This work was supported in part by the United States Department of Energy through the Sandia National Laboratories ASCI VIEWS Data Discovery Program, contract number DE-AC04-76DO00789.

\section{References}

[1] "The location of The Forest CoverType Dataset", $h t$ tp://ftp.ics.uci.edu/pub/machine-learningdatabases/covtype/covtype.data.gz, 1998.

[2] “weka”, http://www.cs.waikato.ac.nz/ml/weka/

[3] J. A. Blackard, "Description of The Forest CoverType Dataset", $\quad h$ ttp://ftp.ics.uci.edu/pub/machine-learningdatabases/covtype/covtype.info, 2001.

[4] G. Cybenko, "Approximation by Superpositions of a Sigmoidal Function", Mathematics of Control, Signals, and Systems, pp. 2:303-314, 1989.
[5] S. A. Eschrich, "Learning From Less: A Distributed Method for Machine Learning ", Dissertation, U. of South Florida, 2003.

[6] S. Fahlman, "The software of the cascade correlation NN", $h$ ttp://www-2.cs.cmu.edu/ sef/sefSoft.htm.

[7] S. Fahlman and C. Lebiere, "The Cascade-Correlation Learning Architecture", Morgan Kaufmann, NIPS, pp. 191-196, 1991.

[8] K. Hornik and M. Stinchcombe and H. White, "Multilayer Feedforward Networks are Universal Approximators", Neural Networks, pp. 2:359-366, 1989.

[9] C. Igel and M. Husken, "IImproving the Rprop Learning Algorithm", Proceedings of the Second Int'l Symp. on Neural Computation, pp. 115-121, Feb. 2000.

[10] A. Lazarevic and Z. Obradovic, "The distributed boosting algorithm", Proceedings of the seventh ACM SIGKDD international conference on Knowledge discovery and data mining, pp. 311-316, 2001.

[11] J. R. Quinlan, "C4.5: Programs for Machine Learning", Morgan Kaufmann Publishers, Inc., pp. 35-43, 1993.

[12] M. Riedmiller and H. Braun, "A direct adaptive method for faster backpropagation learning: The RPROP algorithm", Proceedings of IEEE Int'l Conference on Neural Networks, pp. 586-591, 1993. 